\title{
Electronic Transport Mechanisms in PVDF/ZnO Nanocomposite Films
}

\author{
Bir B. Bohara ${ }^{1 *}$, Ashok K. Batra1, James R. Currie Jr. ${ }^{2}$ \\ ${ }^{1}$ Department of Physics, Chemistry, and Mathematics (Materials Science Group), College of Engineering, Technology and \\ Physical Sciences, Alabama A \& M University, Normal (Huntsville), AL, USA \\ ${ }^{2}$ NASA, Huntsville, AL, USA \\ Email: *bbohara@bulldogs.aamu.edu
}

How to cite this paper: Bohara, B.B., Batra, A.K. and Currie Jr., J.R. (2019) Electronic Transport Mechanisms in PVDF/ZnO Nanocomposite Films. Open Access Library Journal, 6: e5707.

https://doi.org/10.4236/oalib.1105707

Received: August 14, 2019

Accepted: August 30, 2019

Published: September 3, 2019

Copyright $\odot 2019$ by author(s) and Open Access Library Inc.

This work is licensed under the Creative Commons Attribution International License (CC BY 4.0).

http://creativecommons.org/licenses/by/4.0/

\begin{abstract}
In this report, we present fundamental DC conduction mechanisms of polyvinyl difluoride/nano-zinc oxide ( $\mathrm{PVDF} / \mathrm{ZnO})$ composite films. Nanocomposite films of zinc oxide in the PVDF matrix have been fabricated via a solution casting method. Space charge limited conduction (SCLC) was found to be a prominent conduction mechanism in higher voltage region and SchottkyRichardson mechanism was a possible conduction mechanism in a higher temperature region. DC activation energy for composites was found to decrease with increasing concentration of $\mathrm{ZnO}$ nanoparticles and reduced graphene oxide (RGO) with $1.41 \mathrm{eV}$ (9.9 wt\%), $1.04 \mathrm{eV}$ (33.33 wt\%), and $0.95 \mathrm{eV}$ (33.33 wt $\%+$ RGO), respectively.
\end{abstract}

\section{Subject Areas}

Nanometer Materials

\section{Keywords}

PVDF, Nano-Composites, ZnO, DC Conduction, Reduced Graphene Oxide

\section{Introduction}

Charge transport in the variety of polymers has been investigated extensively during the past few decades to understand the nature and characteristics of carriers in these materials [1] [2] [3] [4] [5]. Charge transport in functional polymers and metallopolymers is important and interesting in the view of their applications in organic electronics. Thus, attempts are being made to replace the silicon-based electronic devices with polymer-based devices [5]. Polymers are utilized to manufacture flexible and foldable electronic device such as organic 
solar cells, pyroelectric infrared detectors, organic thin film transistors (OTFTs), Polymer light-emitting diodes (PLEDs) and others [5] [6] [7] [8]. The applicable properties of polymers could be tailored in a controlled way to achieve the desired parameters values and properties to be used in devices via fabrication of polymer composites with embedded suitable nanoparticles [9] [10]. There is consistent demand for new organic material systems for specific applications. This demand dictates material scientists to develop new material systems. The search for new material systems and their applications have led to the design of the variety of "ceramic:polymer" composites. It is obvious that the diversity and specificity in materials properties as required by the modern applications cannot be fulfilled by the single-phase materials [9] [10]. Since the composites contain two or more chemically varied materials or phases, it is possible to tailor electrical, optical, and mechanical properties catering to a variety of applications. Recent studies of ceramic-polymer based composites show potential usefulness via large area, lightweight, enhanced strength, and flexible elements due to polymers [10]. Composites possess hybrid properties derived from individual components. Thus, it is warranted to investigate polymer composites extensively for the mechanisms of charge transport phenomena for their use in organic devices.

Polyvinylidene Fluoride (PVDF) has become appealing to numerous industries for their inexpensive, lightweight, biologically compatible, and mechanically stable structures. It can undertake a large amount of deformation while maintaining ample forces. It has an expeditious response time, very low density, and eminent pliability when compared to electroactive ceramics and shape memory alloys. The piezoelectric PVDF and its copolymers have wide applications in actuators and sensors [11]. These materials are utilized as fibers and films in engineering applications such as piezo-laminated columns, active micro air vehicle wings, and shape correction films in space applications where linear movement is required. Other applications may include proton exchange membranes, filtration membranes, structural health monitoring, thermal and mechanical energy harvesters, endoscopic tactile sensors, and microfluidics control [12].

Zinc oxide $(\mathrm{ZnO})$ is an optically appealing material which responses to strains without poling with striking electronic properties [13]. ZnO possesses the combination of typical properties such as electrical, optical and piezoelectric. Due to these unique properties, it has been extensively utilized in various applications like liquid crystal displays, window coatings, storage capacitors, ultrasonic transducers, gas sensors covering various materials, optoelectronic devices, solar cells, and surface acoustic wave (SAW) devices [12]. Graphene (GR) is a two-dimensional sheet of $\mathrm{sp}^{2}$-bonded carbon atoms packed in a honeycomb crystal lattice. Because of its striking flexibility, high aspect ratio, large specific area, excellent mechanical strength, good electrical and thermal conductivities, it has attracted tremendous attention to researchers. Reduced graphene oxide (RGO) is also an excellent dopant for their use in nanocomposite-based devices. 
To the best of author's knowledge, no work has been reported on DC conduction mechanisms of $\mathrm{PVDF} / \mathrm{ZnO}$ or $\mathrm{PVDF} / \mathrm{ZnO} / \mathrm{RGO}$ nanocomposite films. Therefore, in the present investigation, it was thought worthwhile to understand the nature of charge carriers' transport and trap distribution within these nanocomposites. For this purpose, DC-conduction properties of these composites were studied in detail. The dependence of DC-current on the electric field, temperature, and the concentration of nanoparticles are studied extensively and results are reported in this paper.

\section{Experimental Technique}

The PVDF/ZnO films were fabricated via solution-casting technique. First of all, the suitable amount of PVDF, supplied by Aldrich, was dissolved into methyl-ethyl-ketone (MEK) or dimethylformamide (DMF) at about $60^{\circ} \mathrm{C}$. A requisite amount of nano zinc oxide ( $\mathrm{ZnO} ; 20-30 \mathrm{~nm}$; 99\%) particles, supplied by MTI corporation CA, was then added to form PVDF/ZnO blend. Then, this mixture was mechanically/ultrasonically agitated for several hours to break up the agglomerates to obtain a homogenous mixture. Thus, the obtained solution was then poured into Petri dishes to allow the evaporation of the solvent. Two parallel silver conducting electrodes were deposited on opposite faces of the composite film to form a parallel plate capacitor and then cut into $\sim 10 \mathrm{~mm} \times 10$ $\mathrm{mm}$ sized element for testing. The electroded samples were poled at room temperature with $2 \mathrm{kV}$ voltage for $1 / 2$ hour via corona poling. After the poling process, the samples were short-circuited and annealed at $50^{\circ} \mathrm{C}$ for 1 hour to remove any extrinsic charges injected during poling. A detailed fabrication process of the composite films is schematically presented in Figure 1. Samples of PVDF embedded with increasing concentration of $\mathrm{ZnO}$ nanoparticles/RGO are listed on Table 1, with designated names.

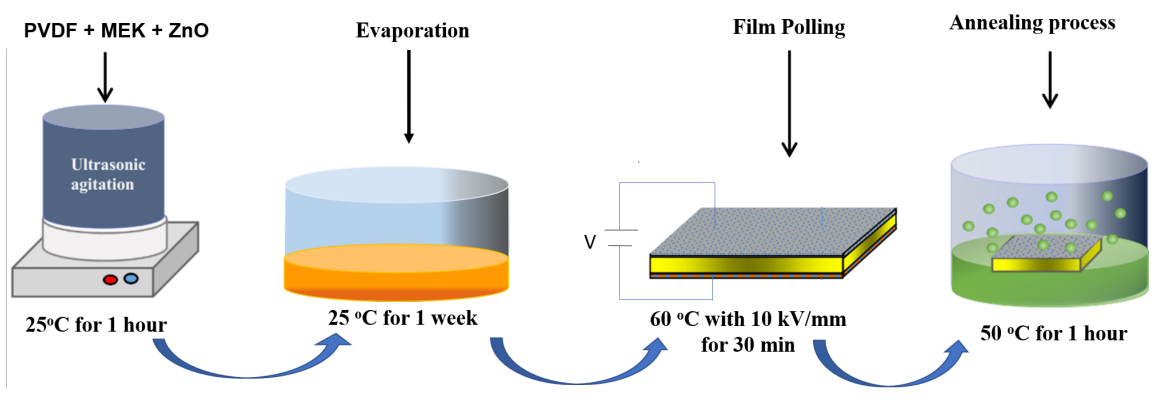

Figure 1. Fabrication process of PVDF/ZnO nanocomposite films.

Table 1. A list of composite films fabricated with various wt $\%$ of $\mathrm{ZnO}$ nanoparticles in PVDF matrix.

\begin{tabular}{ccccc}
\hline Composite films & Mass of PVDF (g) & Mass ZnO (g) & Wt\% of ZnO & RGO \\
\hline Z1 & 1 & 0.1 & 9.10 & - \\
Z3 & 1 & 0.5 & 33.33 & - \\
Z6 & 1 & 0.5 & 33.33 & RGO \\
\hline
\end{tabular}


Isothermal iteration has been carried out in the temperature range from $20^{\circ} \mathrm{C}$ - $80^{\circ} \mathrm{C}$. Drude model can be utilized to measure bulk DC conductivity according to Ohm's law:

$$
\sigma_{d c}=\frac{I t}{V A}
$$

where " $P$ " is the current in ampere and $V$ is the applied voltage in volt over the samples. $A$ and $t$ are area and thickness of the nanocomposite films, respectively. Keithley model 6517 electrometer was utilized to measure two terminal dc current.

\section{Results and Discussions}

\subsection{Steady State Electrical Conduction}

Absorption current is observed in bulk dielectric materials hence the leakage currents are measured after a period at the test temperature of a device under test (DUT) and applied electric field [14]. At the stabilized test temperature of the sample and applied an electric field, the currents were measured after 5 seconds of turning "on" the electrometer. J-V characteristics of PVDF/ZnO composite films at $40^{\circ} \mathrm{C}$ is depicted in Figure 2(a), that was taken at in the voltage region of $0.5 \mathrm{~V}$ to $15 \mathrm{~V}$. The linear nature of J-V curve exhibits the ohmic conduction mechanism in all composite films with positive slope following the power law of the form $J a V^{n}$, where $\mathrm{n}$ is power index. The calculated value of power index, $\mathrm{n}$, ranges from $0.94-0.99$. This indicates the ohmic conduction in composite films and hence an electric conduction is led merely by the resistance of the film materials. It can be depicted from the J-V curve that the current in films decreases with increase in the concentration of $\mathrm{ZnO}$ nanoparticles in the PVDF matrix. Also, it is observed that current in the blend sample embedded with $\mathrm{ZnO}$ and $\mathrm{RGO}$ increases with the concentration of $\mathrm{ZnO}$ in the solution.

Because of dipolar polarization in these samples, the role of dipolar and space charge polarization contribution to conduction mechanisms may be accounted in structurally heterogeneous composite materials. Thus, the other relevant types of conduction mechanisms were also investigated via existing processes and models.

\subsection{Space Charge Limited Conduction (SCLC)}

SCLC mechanism is governed in which charge is injected at the electrode-polymer interface that has a large excess charge carrier density at the injecting electrode causing the SCLC current flow. The space charge limited current density is given by [14]

$$
J=\frac{9}{8} \in \mu \frac{V^{2}}{d^{3}}
$$

where $J$ is the current density of space charge limited conduction at voltage $V$ and $d$ is the thickness of samples. $\in$ and $\mu$ are permittivity and permeability of the material under test. 


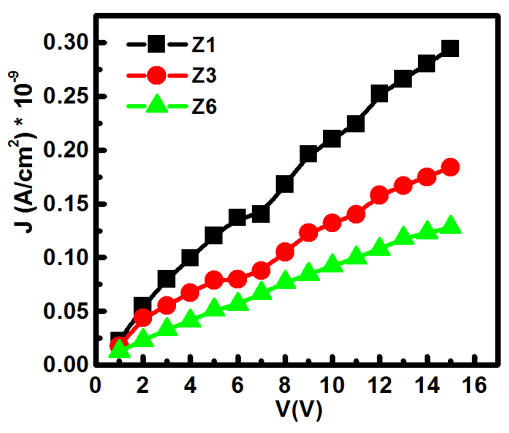

(a)

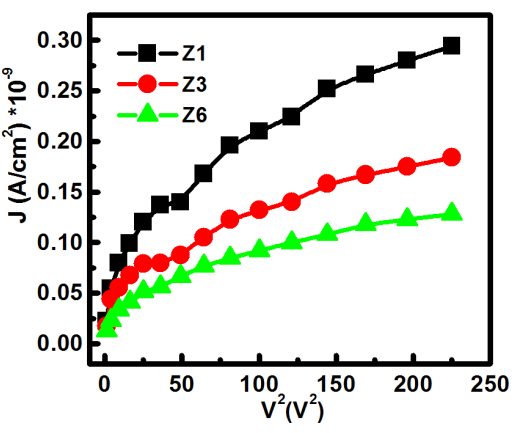

(b)

Figure 2. (a) J-V Characteristics of $\mathrm{PVDF} / \mathrm{ZnO}$ composite films; (b) The current $(J)$ vs. square of applied voltage $\left(\mathrm{V}^{2}\right)$ plots.

From Equation (2), current density $J$ is proportional to $d^{3}$. This relation $J a$ $d^{-m}$, where m represents the slope of the curve and rely upon trap distribution. The value of slope $\mathrm{m}<3$ indicates the probability of PF or Schottky mechanism, whereas, $m \geq 3$ connotes the possibility of SCLC mechanism [15] [16]. From Figure 2(b), however, overall nature of the curve is non-linear indicating the non-existence of SCLC mechanism, the linear relationship at higher voltages ( $>10$ Volts) of $\mathrm{J}^{-} \mathrm{V}^{2}$ curves for $\mathrm{PVDF} / \mathrm{ZnO}$ nanocomposite films indicates the dominance of the SCLC conduction.

\subsection{Tunneling or Fowler-Nordheim Mechanism}

In case of very thin samples or samples that contain the substantial number of defects tunneling process comes into play [16]. This phenomenon is characterized by the current generation without the transport of charge carriers in the conduction band or in the valence band. The Fowler-Northeim relation for Jcan be expressed as:

$$
\log \frac{J}{V^{2}}=\log A-\frac{\varphi}{V}
$$

where $A=\frac{q^{3}}{8 \pi h \varphi_{B}}, \varphi=\frac{8 \pi\left(2 q m_{T}^{*}\right)^{1 / 2}}{3 h} \varphi_{B}^{2 / 3}$, and $J, V, q, h, m_{T}^{*} \varphi_{B}$ are current density, applied voltage, electronic charge, Plank's constant, tunneling effective mass and barrier potential, respectively.

If the nature of the $\log \mathrm{J} / \mathrm{V}^{2}$ vs. $1 / \mathrm{V}$ plot is linear with negative slope, then the Fowler-Nordheim tunneling is prevalent. Since our investigated samples are thick, the tunneling is not likely expected. It is observed in Figure 3(a) that plots have non-linear characteristic and do not possess negative slope which ruled out the presence of tunneling effect in $\mathrm{PVDF} / \mathrm{ZnO}$ composites.

\subsection{Poole-Frenkel (PF) Conduction}

The current density $J$ is generated because of the thermal excitation of trapped electrons or holes by the applied field in Poole-Frenkel conduction [17] [18]. PF conduction is also known as internal Schottky thermal emission or field-assisted 


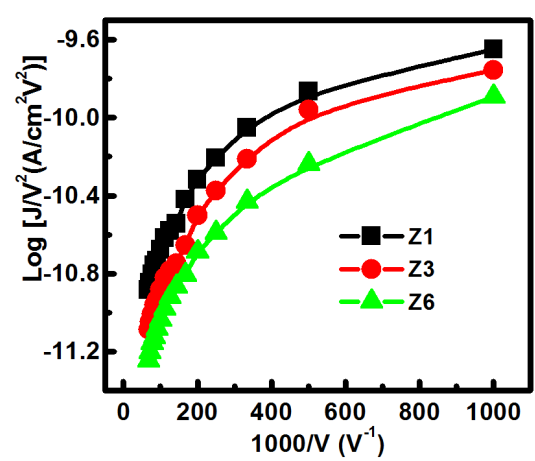

(a)

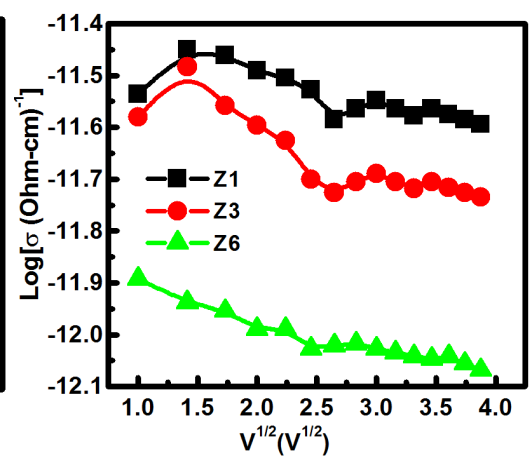

(b)

Figure 3. (a) Fowler-Nordheim plots of $\mathrm{PVDF} / \mathrm{ZnO}$ composite films at $40^{\circ} \mathrm{C}$; and (b) Poole-Frenkel (PF) plots for PVDF/ZnO composite films at $40^{\circ} \mathrm{C}$.

thermal ionization. The bulk-limited electric transport emerges due to the emission of electrons/holes from trapping centers in the film samples due to the effects of temperature and electric field. The Poole-Frenkel field-depended conductivity is given by equation [15]:

$$
\log \sigma=\log \sigma_{0}+\frac{\beta_{P F}}{2 k T} F^{1 / 2}
$$

where $\beta_{P F} F, k, T$ are the Poole-Frenkel coefficient, static electric field, Boltzmann constant, and absolute temperature, respectively.

The linear nature of $\log \sigma$ vs $F^{1 / 2}$ plots with positive slope indicates $P F$ conduction. $P F$ plot (Figure 3(b)) of PVDF/ZnO shows nonlinear nature for all composite films and neither positive slope nor negative which excludes the possibility of $P F$ conduction in the PVDF/ZnO films. This may cause because of the presence of space charges accumulated near the electrodes creating non-uniform field distribution between the electrodes.

\subsection{Schottky-Richardson Mechanism}

The interaction of an electric field at a metal-polymer interface with the image force reduces the potential barrier in Schottky type conduction mechanism. The Schottky-Richardson model current-voltage relationship is expressed by the equation:

$$
\log J=\log A T^{2}-\frac{\varphi}{k T}+\beta_{s} F^{\frac{1}{2}},
$$

where $\beta_{s}=\frac{q \sqrt{q / 4 \pi \varepsilon_{r} \varepsilon_{0}}}{k T}$; and $F, A, T, \varphi k, q, \varepsilon_{r}, \varepsilon_{0}$ are an electric field, effective Richardson constant, absolute temperature, Schottky barrier height, Boltzmann constant, electronic charge, optical dielectric constant, and permittivity in vacuum, respectively.

Figure 4(a) represents the Schottky plot of investigated samples showing nonlinearity with positive slopes. If the linear nature of $\log J-\mathrm{V}^{1 / 2}$ curve accompanies a positive slope, then the existence of Schottky-Richardson mechanism is 
confirmed. It is observed that the nature of the curve is linear and have a positive slope at higher voltages. Space charges accumulated near the electrodes, producing non-uniformity of field distribution between electrodes, might be the probable cause of the deviation. The linear curve at higher voltages indicates to electronic-type conduction that may be caused by either Schottky emission mechanism or Poole-Frenkel mechanism [19]. Figure 4(b) shows the Richardson line, $\log \left(\mathrm{J} / \mathrm{T}^{2}\right)$ vs. 1000/T plot, which shows nearly linear lines with a negative slope for all samples, showing the relevance of the Schottky-Richardson conduction.

Figure 5(a) shows the temperature dependency of the current density in which $\log J$ is linearly increasing with temperature. Furthermore, the absence of any thermodynamic transition in the selected temperature range is indicated by sharp linearity at higher temperature region.

Figure 5(b) depicts the temperature dependence of dc conductivity of $\mathrm{PVDF} / \mathrm{ZnO}$ composite samples. It is observed that the dc conductivity of $\mathrm{PVDF} / \mathrm{ZnO}$ nanocomposite samples decreases with increasing the concentration of $\mathrm{ZnO}$ and RGO nanoparticles. Our result corresponds with the Arrhenius relationship that expresses temperature dependence of dc conductivity and given by following equation [19]:

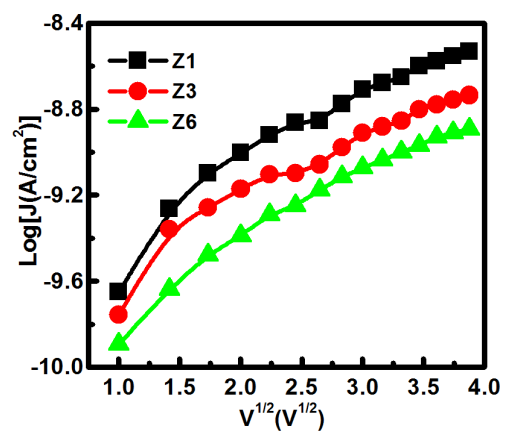

(a)

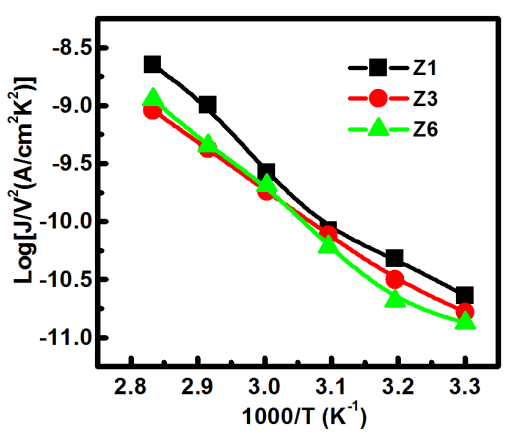

(b)

Figure 4. (a) Schottky plots of $\mathrm{PVDF} / \mathrm{ZnO}$ nanocomposite samples at $40^{\circ} \mathrm{C}$; (b) Richardson plots of $\mathrm{PVDF} / \mathrm{ZnO}$ nanocomposite films at the applied voltage of $5 \mathrm{~V}$.

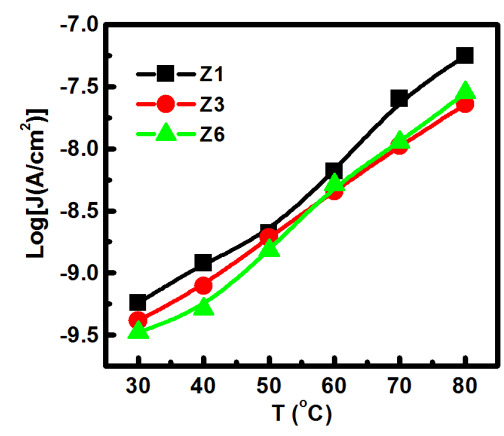

(a)

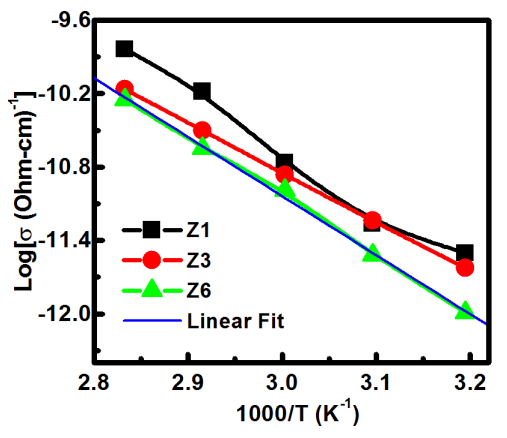

(b)

Figure 5. (a) Current density vs. temperature plot of PVDF/ZnO nanocomposite films at the applied voltage of 5 volts; (b) temperature dependence of dc conductivity of PVDF/ZnO composite films. 


$$
\sigma_{d c}=\sigma_{0} \exp \left(-\frac{\Delta E}{k_{b} T}\right)
$$

where $\Delta E, k_{b}, \sigma_{\mathrm{o}}$ represent the thermal activation energy, Boltzmann's constant $\left(8.617 \times 10^{-5} \mathrm{eV} / \mathrm{K}\right)$, and pre-exponential factor/maximum conductivity, respectively.

It is obvious that the slopes of $\mathrm{PVDF} / \mathrm{ZnO}$ samples are negative with linear plots. Activation energy for composite samples Z1 (9.10 wt\%), Z3 (33.33 wt\%), and Z6 (33.33 wt\% + RGO) was $1.41 \mathrm{eV}, 1.04 \mathrm{eV}$, and $0.95 \mathrm{eV}$, respectively. One can observe dc conductivity activation energies decreasing with increasing wt\% of $\mathrm{ZnO}$ and $\mathrm{RGO}$.

\section{Conclusion}

$\mathrm{PVDF} / \mathrm{ZnO}$ nanocomposite films have been fabricated via a solution casting method. The fabricated films were characterized for their dc electronic conduction phenomena. DC transport investigation indicates the prominent Schottky-Richardson mechanism at higher temperature region with SCLC conduction at high voltage region. Activation energy was found to be decreasing with increasing concentration of $\mathrm{ZnO}$ and $\mathrm{RGO}$.

\section{Acknowledgements}

The authors gratefully acknowledge support for this work through the National Science Foundation grant \#RISE-HRD 1546965. Special thanks to Prof. M. D. Aggarwal for his support in our research. Financial assistance from AAMU-Title III program is appreciated.

\section{Conflicts of Interest}

The authors declare no conflicts of interest regarding the publication of this paper.

\section{References}

[1] Nalwa, H.S. (1995) Ferroelectric Polymers. Marcel Dekker, New York. https://doi.org/10.1201/9781482295450

[2] Nayak, H.C. (2013) Study of Conduction Mechanism in Poly(9-Vinylcarbozole) Oure and Doped with Ferrocene. International Journal of Scientific and Engineering Research, 4, 1228-1234.

[3] Martin, R. and Bhuiyan, A.H. (2011) Electrical Transport Mechanism in Plasma Polymerized 2, 6, Diethylaniline Thin Films. Thin Solid Films, 51, 3462-3467. https://doi.org/10.1016/j.tsf.2010.12.240

[4] Abd-El Kader, F.H., Osman, W.H. and Hafez, R.S. (2013) DC Conduction Mechanism and Dielectric Properties of Poly(methacrylate)/Poly(vinyl acetate) Blends Doped and Undoped with Malachite Green. Physica B, 408, 140-150. https://doi.org/10.1016/j.physb.2012.09.027

[5] Deepak, K., Roy, A., Anjaneyulu, P., Kandaiah, S. and Pinjare, S.L. (2017) Charge Transport Mechanism in p-Type Copper Ion Containing Triazine Thiolate Metal- 
lopolymer Thin Film Devices. Journal of Applied Physics, 122, Article ID: 164504. https://doi.org/10.1063/1.4993983

[6] Batra, A.K., Aggarwal, M.D., Edwards, M.E. and Bhalla, A. (2008) Present Status of Polymer: Ceramic Composites for Pyroelectric Infrared Detectors. Ferroelectrics, 366, 84-121. https://doi.org/10.1080/00150190802363207

[7] Paik, H., Choi, Y., Hong, S. and No, K. (2015) Effect of Ag Nanoparticle Concentration on Electrical and Ferroelectric Properties of Ag-P(VDF-TRFE) Composite Films. Scientific Reports, 5, Article No. 13209. https://doi.org/10.1038/srep13209

[8] Batra, A.K. and Motasim, A. (2017) Power Harvesting via Smart Materials. SPIE Press, Bellingham. https://doi.org/10.1117/3.2268643

[9] Ram, R., Rahaman, M. and Khastgir, D. (2015) Electrical Properties of PVDF/Multi-Walled Carbon Nanotubes (MWCNT) Semi-Transparent Composites: Modelling of DC Conductivity. Composites Part A: Applied Science and Manufacturing, 69, 30-39. https://doi.org/10.1016/j.compositesa.2014.11.003

[10] Aggarwal, M.D., Batra, A.K., Guggilla, P., Edwards, M.E., Penn, B. and Currie, J.R. (2010) Pyroelectric Materials for Uncooled Infrared Detectors. NASA TM, 251-258.

[11] Puertolas, J.A., Garcia-Garcia, J.F.F., Pascul, J. and Gonzalez-Dominguez, J.M. (2017) Dielectric Behavior and Electrical Conductivity of PVDF Filled with Functionalized Single-Walled Carbon Nanotubes. Composites Science and Technology, 152, 263-274. https://doi.org/10.1016/j.compscitech.2017.09.016

[12] Batra, A.K. and Aggarwal, M.D. (2013) Pyroelectric Materials. SPIE Press, Bellingham.

[13] Öğüt, E., Sinan Yördem, O., Menceloğlu, Y.Z. and Papila, M. (2007) Poly(vinylidene fluoride)/Zinc Oxide Smart Composite Material, Behavior and Mechanics of Multifunctional and Composite Materials 2007. Proceedings of SPIE, Vol. 6526, 65260Q. https://doi.org/10.1117/12.717703

[14] Du, H., Liang, W., Gao, M., Zhang, Y., Chen, C. and Lin, Y. (2015) Leakage Properties of $\mathrm{BaTiO}_{3}$ Thin Films on Polycrystalline Ni Substrates Grown by Polymer-Assisted Deposition with Two-Step Annealing. Journal of Alloys and Compounds, 642, 166-171. https://doi.org/10.1016/j.jallcom.2015.04.025

[15] Kamal, M.M. and Bhuiyan, A.H. (2014) Direct Current Electrical Conduction Mechanism in Plasma Polymerized Pyrrole Thin Films. Journal of Modern Science and Technology, 2, 1-9.

[16] Yang, B.L., Lai, P.T. and Wong, H. (2004) Current Conduction Mechanism in Thin Gate Dielectrics Microelectron. Reliability, 44, 709-718. https://doi.org/10.1016/j.microrel.2004.01.013

[17] Batra, A., Bohara, B., Mills, J., Wright, R. and Kenney, B. (2017) Mechanisms of DC Conduction in Smart PMN-PT/Paint Nanocomposite Films. Journal of Materials Science: Materials in Electronics, 28, 13336-13336. https://doi.org/10.1007/s10854-017-7170-5

[18] El Tayyan, A.A. and Khogali, A. (2004) DC Conduction in $\mathrm{Fe}^{3+}$ Poly(9-vinylcarbazole) Doped Films. Chinese Journal of Physics, 42, 392-397.

[19] Khissi, M., El Hasnaoui, M., Belattar, J., Graca, M.P.F., Achour, M.E. and Costa, L.C. (2011) DC Electrical Conductivity Studies on Copolymer/Carbon Black Composites. Journal of Materials and Environmental Science, 2, 281-284. 